\title{
ARTICULAÇÃO ENSINO-SERVIÇO E VIGILÂNCIA DA SAÚDE: A PERCEPÇÃO DE TRABALHADORES DE SAÚDE DE UM DISTRITO ESCOLA
}

\author{
TEACHING-SERVICE AND HEALTH SURVEILLANCE INTEGRATION: A SCHOOL DISTRICT'S \\ HEALTH WORKERS' PERCEPTION
}

\author{
Juliana Guisardi Pereira ${ }^{1}$ \\ Lislaine Aparecida Fracolli ${ }^{2}$
}

Resumo Para ser colocada em prática, a Vigilância da Saúde necessita que os trabalhadores de saúde estejam preparados para operacionalizá-la. O objetivo deste artigo é descrever a percepção de profissionais de saúde sobre as contribuições da articulação ensino-serviço para a implantação da Vigilância da Saúde. Trata-se de uma pesquisa qualitativa, realizada no Distrito de Saúde Escola Butantã, no município de São Paulo. Foram sujeitos da pesquisa médicos e enfermeiros que atuavam nas unidades de saúde da região. Os dados foram coletados mediante entrevistas e analisados segundo a técnica do discurso do sujeito coletivo. Os resultados mostraram que, segundo os profissionais de saúde, a articulação ensino-serviço se efetiva pela supervisão dos alunos de graduação em Medicina e Enfermagem, no cotidiano das unidades de saúde. Para os profissionais de saúde, a contribuição da articulação ensino-serviço para a Vigilância da Saúde é pequena e se resume às atividades curriculares dos alunos. Falta um projeto político do município para a Vigilância da Saúde, e a academia poderia contribuir na sua elaboração. Conclui-se que, para a mudança das práticas assistenciais, é importante a construção em parceria, entre gestores, trabalhadores, docentes e alunos, de um projeto político-pedagógico.

Palavras-chave Vigilância da Saúde; integração docente-assistencial; educação em saúde.
Abstract To be put into practice, Health Surveillance needs health workers to be prepared in order to operationalize it. The purpose of this article is to describe the perception health professionals have on the contributions of the teaching-service integration for the deployment of Health Surveillance. This is a qualitative study conducted in the Butantã School Health District, in the municipality of São Paulo. Research subjects were physicians and nurses working for health facilities located in the region. Data was collected through interviews and analyzed pursuant to the collective subject discourse technique. The results showed that, according to the health professionals, the teaching-service integration is carried out through the supervision of undergraduate medical and nursing students in routine health facility activities. To the health professionals, the contribution made by the teaching-service integration for Health Surveillance is small and boils down to the students' curricular activities. There is a need for a municipal political project for Health Surveillance, and the academy could assist in its preparation. The conclusion is that in order for there to be change in the care practices, it is important to build a political-pedagogical project in partnership with managers, workers, teachers, and students.

Keywords Health Surveillance; teaching-care integration, health education. 


\section{Introdução}

O termo 'vigilância da saúde' começou a ser utilizado no início da década de 1990, no contexto da implantação do Sistema Único de Saúde (SUS), um momento em que se discutia a necessidade de reorientação e reorganização dos serviços. A Vigilância da Saúde apontava para o entendimento dos problemas de saúde e das condições de vida em sua totalidade, buscando compreender e intervir nos determinantes e condicionantes do processo saúde-doença (Bertolozzi e Fracolli, 2004).

Numa análise bibliográfica sobre o conceito de Vigilância da Saúde, Faria et al. (2005) constataram que alguns autores relacionam o termo à ampliação do objeto da Vigilância Epidemiológica; outros, à proposta de articulação entre Vigilância Epidemiológica e Vigilância Sanitária; outros, ainda, à proposição de uma organização dos processos de trabalho, incorporando a promoção da saúde e a intersetorialidade por meio de ações territoriais. Este estudo se apoia nessa última concepção, que referencia a Vigilância da Saúde à organização de serviços, correspondendo, dessa forma, a um modelo assistencial, ou seja, a um conjunto de saberes e práticas para viabilizar as diretrizes teórico-políticas do SUS.

Para Teixeira, Paim e Vilasbôas (1998), ao lado de modelos alternativos, coexistem modelos assistenciais diversos no cenário da saúde brasileira, com predominância de dois grandes modelos hegemônicos: o médico-assistencial e o sanitarista. Esses autores entendem por modelo médico-assistencial aquele pautado no atendimento médico voltado para o controle e o tratamento de doenças, dirigido ao sujeito individual e que se utiliza, como meios de trabalho, dos conhecimentos e tecnologias para diagnóstico e terapêutica de doenças.

Já o modelo sanitarista tem como objeto os modos de transmissão e os fatores de risco das doenças e utilizam, como meios de trabalho, a educação em saúde, o saneamento, o controle de vetores, entre outros. Ainda segundo esses autores, o modelo de Vigilância da Saúde estende o objeto das ações de saúde às determinações sociais que afetam os distintos grupos sociais e que dependem de suas condições de vida.

O modelo de Vigilância da Saúde inclui, entre os seus meios de trabalho, tecnologias de comunicação social que estimulam a participação dos grupos na promoção e defesa de suas condições de vida e saúde, além de propor a incorporação da população organizada, como sujeito das ações de saúde, em parceria com os trabalhadores de saúde (Pereira, 2007). Assim, é possível compreender que o modelo de Vigilância da Saúde incorpora e supera os modelos anteriormente propostos, redefinindo o objeto, os meios e a forma de organização do trabalho. A operacionalização desse modelo assistencial depende muito da transformação das lógicas que presidem o trabalho e a formação de profissionais de saúde. 
Desde 2004, o Ministério da Saúde vem discutindo o fato de as instituições formadoras terem reproduzido modelos de ensino conservadores, centrados na fisiopatologia, na anátomo-clínica, em procedimentos e equipamentos de apoio diagnóstico e terapêutico e limitados à aprendizagem no hospital universitário. E ressalta que a formação não deve centrar-se apenas na busca eficiente de evidências no diagnóstico, cuidado, tratamento, prognóstico, etiologia e profilaxia das doenças e agravos, devendo comprometerse com o desenvolvimento de condições de atendimento às necessidades de saúde das pessoas e das populações e com a gestão setorial e o controle social em saúde, redimensionando o desenvolvimento da autonomia das pessoas até a condição de influência na formulação de políticas do cuidado (Brasil, 2004).

A questão do redirecionamento da formação de profissionais de saúde para o SUS tomou nos últimos anos dimensão de política pública. Várias políticas públicas na área de saúde têm sido criadas a fim de possibilitar que o ensino das profissões da saúde possa fundamentar-se na construção do conhecimento a partir da realidade e na participação ativa do estudante no processo ensino-aprendizagem, bem como no seu envolvimento com a transformação da mesma (Ceccim e Feuerwerker, 2002).

Nos últimos anos, o Ministério da Saúde, em parceria com o Ministério da Educação (MEC), vem propondo projetos que estimulam a parceria entre universidades e serviços de saúde, com o intuito de garantir um ensino mais aproximado da realidade. Tais projetos têm como foco o fortalecimento do SUS e de seus princípios. Dentro desse grupo de projetos, podem ser citados, principalmente, o Programa de Incentivo à Mudança Curricular nos Cursos de Graduação em Medicina (Promed), de 2002; o projeto Vivências e Estágios na Realidade do Sistema Único de Saúde do Brasil (VER-SUS/Brasil), de 2004; os programas Integralidade e Aprender-SUS, também de 2004; e o Programa Nacional de Reorientação da Formação Profissional em Saúde (Pró-Saúde), de 2005 (Pereira, 2007).

A grande aposta que existe no cenário brasileiro é a de que, com a mudança no foco da formação dos profissionais de saúde e com a inserção dos processos de ensino-aprendizagem no interior dos serviços de saúde conseguir-se-á realizar duas mudanças essenciais para o SUS: alterar os processos de trabalho ao mesmo tempo em que se altera o paradigma flexneriano da formação em saúde.

Reconhecendo os esforços despendidos pela sociedade, como um todo, e por docentes e técnicos de saúde, de um modo mais especifico, esta pesquisa resolveu investigar como ocorre a articulação ensino-serviço para a operacionalização da Vigilância da Saúde. O objetivo deste artigo é descrever, na percepção dos trabalhadores de saúde, a articulação ensino-serviço e a sua contribuição para o modelo de Vigilância da Saúde. 


\section{Metodologia}

Esta é uma pesquisa de abordagem qualitativa, tipo estudo de caso, descritivo e exploratório. O cenário de estudo tomou como foco os processos de ensino-aprendizagem realizados no Distrito Escola do Butantã, localizado na região oeste do município de São Paulo, São Paulo. Tal escolha se justificou pelo fato de, há muitos anos, a região ter estabelecido uma parceria com a Secretaria Municipal de Saúde e a Universidade de São Paulo (USP) - principalmente por meio da Faculdade de Medicina (FMUSP), da Escola de Enfermagem (EEUSP) e da Faculdade de Saúde Pública (FSPUSP) - para atuação conjunta na gestão, ensino e pesquisa em saúde na região. Partiu-se da suposição de que o Distrito Escola do Butantã corresponderia a uma iniciativa mediante a qual seria possível 'desenhar' um modelo de Vigilância da Saúde pautado na intersecção entre debates e proposições de docentes da universidade e de profissionais dos serviços de saúde, de forma a conferir melhor qualidade à Vigilância da Saúde, com a integração de pesquisa, ensino e assistência num território.

Foram sujeitos desta pesquisa cinco trabalhadores de saúde que atuavam na Estratégia Saúde da Família (ESF) no Distrito Escola do Butantã. Esses trabalhadores atuavam como preceptores de ensino e recebiam alunos de graduação (dos cursos de Medicina e Enfermagem) no cotidiano do trabalho. Os sujeitos da pesquisa foram um gerente (da Unidade Básica de Saúde - UBS), dois médicos da equipe de Saúde da Família, um médico sanitarista e um enfermeira da equipe de Saúde da Família.

Os dados foram coletados por meio de entrevistas semiestruturadas. Os sujeitos foram entrevistados, na maioria dos casos, nas unidades de saúde, durante o horário de trabalho. As entrevistas buscavam identificar qual o conhecimento dos profissionais acerca das disciplinas e dos programas de ensino ali desenvolvidos. Procuravam identificar, também, o quanto eles participavam da formulação desses programas de ensino e em que medida o serviço incorporava os conteúdos e as proposições feitas por ocasião das atividades dos alunos nas unidades de saúde. As entrevistas foram gravadas e transcritas.

$\mathrm{Na}$ análise, utilizou-se a técnica do discurso do sujeito coletivo (Lefrève e Lefrève, 2000). O discurso do sujeito coletivo (DSC) configura uma 'coletividade discursiva' cujo conteúdo está composto pelo que é falado por um sujeito individual e pelo o que o seu 'companheiro de coletividade' atualiza por ele. Os discursos são submetidos a uma análise de conteúdo, que se inicia pela sua decomposição nas principais ancoragens, ou ideias centrais, presentes em cada um, individualmente, e na reunião de todos. Segue-se a isso uma síntese, que visa à reconstituição discursiva da representação social. Os passos até a síntese, segundo Lefrève e Lefrève (2000), incluem leitura 
do conjunto dos depoimentos coletados nas entrevistas; leitura de cada pergunta em particular e resposta a ela, marcando-se as expressões-chave selecionadas; identificação das ideias centrais de cada resposta; e análise de todas as expressões-chave e ideias centrais, agrupando-se as semelhantes em conjuntos homogêneos. Posteriormente, procede-se à identificação e à nomeação da ideia central do conjunto homogêneo, à construção dos DSC e à atribuição de um nome, tema ou identificação para cada um dos DSC.

Esta pesquisa, em conformidade com a resolução n. ${ }^{\circ}$ 196/1996, foi aprovada pelo Comitê de Ética em Pesquisa da Secretaria Municipal da Saúde do município de São Paulo (CEP/SMS). Para a coleta de dados, foi utilizado um termo de consentimento livre e esclarecido, sendo garantido todo o sigilo de forma a assegurar a privacidade dos sujeitos.

\section{Resultados e discussão}

Com base nos discursos dos trabalhadores de saúde foi possível descrever as percepções que eles possuíam sobre a articulação da universidade com os serviços de saúde do Distrito Escola de forma geral e, mais especificamente, em relação ao seu trabalho. Foi possível ainda descrever como eles viam a contribuição (ou não) da articulação com a universidade para a melhoria qualitativa do seu processo de trabalho.

Quanto à percepção dos trabalhadores sobre a articulação ensino-serviço, os discursos nos remeterem a três grandes temas:

\section{Tema 1 - Existe integração ensino-serviço, porém de forma passiva por parte dos profissionais do serviço.}

Os docentes contribuem muito quando os trabalhadores realizam pesquisa e apresentam seus resultados. No ano passado, as alunas fizeram um trabalho de caracterização das famílias da área, aplicaram algumas entrevistas e detectaram problemas. Depois, elaboraram uma tabela com dados estatísticos, que apresentaram numa reunião, mostrando a opinião de alguns usuários sobre os serviços e os problemas mais presentes na comunidade. Foi um trabalho mais dos alunos mesmo. Nos estágios, os alunos chegam com o roteiro pronto. As enfermeiras do serviço estão pouco apropriadas dos objetivos dos estágios e não têm condições de sugerir ou de articular iniciativas. Na Enfermagem, os alunos fazem visitas domiciliárias com os agentes comunitários e trazem um retorno disso - trouxeram inclusive propostas que estão sendo implementadas. Há uma enfermeira na UBS que faz a interface com a Escola de Enfermagem: ela planeja as atividades dos alunos e prepara o 
caminho para o estágio, envolvendo os enfermeiros para o entendimento dos objetivos dos estágios.

A análise do DSC presente nesse tema mostra que as concepções sobre a articulação ensino-serviço que perpassam os discursos dos trabalhadores são diversas e contrárias à ideia de integração, uma vez que estão apoiadas em perspectivas que apontam subalternidade e não parceria. Para alguns profissionais, a integração depende da ação da academia, que traz um programa de ensino pronto, com um roteiro de atividades a ser desenvolvido pelo aluno, e apenas se utiliza do serviço de saúde e do processo de trabalho das equipes para desenvolver a sua proposta. As percepções presentes no DSC mostram ausência de construção conjunta do programa de ensino e das atividades do aluno.

\section{Tema 2 - A integração ocorre mediante a inserção dos alunos na rotina da UBS.}

Os dois cursos procuram vir, entender um pouquinho o que é o trabalho, como é a comunidade, captam problemas e propõem intervenções. $\mathrm{Na}$ Medicina, o primeiro ano tem contato maior com as agentes comunitárias quando conhecem o território e os mecanismos utilizados pelos agentes comunitários para acompanharem a comunidade. Na Enfermagem, os alunos vão para as escolas, fazem grupos, trabalham com a comunidade, que é uma coisa que a unidade também faz. Procura-se planejar para não haver colisão entre os programas da Enfermagem e os da Medicina. A Enfermagem já desenvolveu um jeito de fazer que tem transcorrido tranquilamente, não onera o serviço e não atrapalha a rotina do trabalho. A Faculdade de Medicina interfere nas atividades dos profissionais porque os grupos são muito grandes. Na Medicina, numa fase anterior à vinda dos alunos, são feitas reuniões das quais participam os professores da faculdade e os médicos de família para opinar. Nas reuniões técnicas que ocorrem no posto, quando médicos, enfermeiros, auxiliares e agentes comunitários se reúnem, procura-se levar essas discussões e propostas da faculdade e acolher também a opinião do pessoal: é possível, não é possível, vai atrapalhar, não vai atrapalhar.

A leitura do DSC referente ao tema 2 mostra que para alguns profissionais a integração ensino-serviço está presente quando os alunos se propõem a acompanhar as atividades da equipe sem interferir na dinâmica da UBS.

A análise das percepções presentes nos DSC sobre integração ensinoserviço na saúde evidenciou uma fragilidade no que tange ao trabalho cooperativo que se esperava encontrar entre a universidade e os serviços de atenção 
básica à saúde. Esperava-se que essa articulação resultaria na prestação de uma assistência à saúde diferenciada e inovadora, com os professores ensinando e supervisionando os estudantes em modelos de assistência avançados e em consonância com os princípios do SUS. Na prática, o profissional de saúde não transforma seu processo de trabalho e continua realizando suas ações rotineiras. Contudo, durante o período em que o aluno está no serviço, o profissional realiza outras ações para atender o projeto de ensino dos alunos.

\section{Tema 3 - A integração ocorre de forma diferente entre os profissionais envolvidos, a depender do perfil para o ensino, o qual é avaliado no momento da contratação.}

Cada profissional tem seu envolvimento no projeto de ensino. É lógico que tem de ter vocação: quem não gosta não vai se dar bem aqui. A universidade acabou intervindo na seleção dos profissionais por meio de uma comissão mista entre a universidade e a Secretaria Municipal de Saúde. Assim, fica claro para o profissional o papel de incorporar o ensino nos serviços, pois se avalia, nesse momento, o perfil para a área acadêmica. Já se deixa bem claro que aqui se recebem alunos e, portanto, há um ônus a mais no trabalho, que é a questão do ensino.

Outra ideia que surge no DSC classificado como tema 3 refere-se ao fato de a integração depender do perfil do profissional do serviço, que pode ou não ter vocação para o ensino.

Alguns momentos de intersecção serviço/universidade foram apontados nos discursos, tais como: contratação de profissionais com perfil docente, interface da enfermeira com a Escola de Enfermagem, participação dos médicos de família e dos demais funcionários em reuniões preparatórias para opinar nas atividades práticas dos alunos, discussão de casos e implementação de propostas na UBS.

Foi possível observar, também, um movimento de fortalecimento da articulação ensino-serviço a partir da contratação futura de profissionais para atuar nas unidades básicas que possuam perfil para o ensino, um importante fator para a aproximação das instituições de ensino e de serviço.

Uma maneira de se 'formar' o perfil para o ensino seria a elaboração, pelas instituições de ensino superior, de projetos que incorporem os pressupostos do Programa de Educação pelo Trabalho para a Saúde (PET-Saúde). Esse tipo de programa tem sido assinalado como facilitador na articulação ensino-serviço de forma a propiciar o fortalecimento da Vigilância da Saúde, tanto no que se refere à prática dos serviços de saúde quanto ao preparo de futuros profissionais para o atendimento condizente com seus pressupostos (Brasil, 2010). 
Com relação à contribuição da articulação ensino-serviço para a construção do modelo da Vigilância da Saúde no território, os DSC foram classificados em dois temas, que podem ser considerados quase que percepções antagônicas do mesmo fenômeno, apresentados a seguir.

Tema 1 - A universidade participa da implantação do modelo de Vigilância da Saúde, com a criação de ações na UBS e a atualização dos profissionais da rede.

É mais a Enfermagem que contribui com ações para a implantação da Vigilância da Saúde, por meio de questões conceituais, como a identificação da realidade local, pelos trabalhos elaborados pelos alunos, que problematizam a realidade e trazem a discussão para a unidade. Segundo os enfermeiros que participam das conversas de final de estágio com o docente, enriquece, abre um pouco a cabeça e permite um novo olhar sobre o trabalho. A universidade acabou trazendo capacitações para os agentes comunitários, por meio de aulas ou atividades de campo, com a investigação de doenças como tuberculose, dengue, HIV ou doenças sexualmente transmissíveis. Dessa forma, contribuiu para atualizar até mesmo algumas condutas do médico e do enfermeiro na unidade de saúde. Então, é um aspecto muito amplo, pois levantam a questão epidemiológica, os agravos à saúde, as complicações. A Medicina tem feito menos, porque o eixo principal da intervenção não é discutir modelo.

\section{Tema 2 - A participação da universidade na implementação do modelo da Vigilância da Saúde tem sido pequena por falta de projeto político.}

A participação da universidade tem sido pequena, principalmente por causa da prefeitura. Não se conseguiu dar essa direcionalidade às ações de saúde, embora pesem as ações de vigilância à saúde, mas ela ainda é vista como um sistema específico, com suas atribuições próprias, seja de controle de doenças ou de controle ambiental. Há um buraco na própria prefeitura para conseguir fazer isso. A criação pela prefeitura de São Paulo da Coordenação de Vigilância em Saúde (Covisa) é recente, e, às vezes, duvida-se de se as próprias pessoas que trabalham lá têm clareza do seu trabalho; imagina então nos outros segmentos. A Covisa precisa ampliar sua atuação: está muita fechada na questão da vigilância epidemiológica. O objetivo é ter um serviço cuja lógica seja a de utilizar os dados no planejamento. No momento, isso não é feito - e a coordenação também não faz. Não se tem clareza do conceito e não se discute uma proposta da missão da unidade básica. Responde-se apenas a uma demanda que chega, não à que está por trás dos índices de mor- 
bidade, de mortalidade e dos coeficientes. A lógica do serviço não está organizada na lógica da promoção da saúde; ainda está centrada no modelo hegemônico. Essa discussão dos determinantes e condicionantes do processo saúde-doença é uma discussão ampliada que precisa ser feita na intersetorialidade. Essa conversa, no entanto, é dificílima: um não entende o que o outro está falando. O que acontece hoje é que a Medicina pensa por um lado, a Enfermagem por outro e a prefeitura por outro, e aí todo mundo quer desenvolver isso no embate. Só que a unidade não dá conta de toda essa articulação, por isso é necessário criar um fórum de organização para viabilizar todas essas estratégias.

Quanto à participação da universidade na implantação do modelo da Vigilância da Saúde por meio da integração ensino-serviço, os discursos assinalaram que essa participação é limitada e está circunscrita ao desenvolvimento de ações e à capacitação dos profissionais da rede, notadamente em temas relacionados à vigilância epidemiológica. Os trabalhadores mencionam também a necessidade de iniciativa da Secretaria Municipal de Saúde visando propiciar a mudança do modelo assistencial no município com base na construção de nova lógica assistencial.

A saúde no município de São Paulo tem uma história bastante complexa. Até a década de 1980, a saúde no município desenvolveu-se direcionada à assistência individualizada e orientada para a clínica, com destaque para a atenção materno-infantil. Ainda nessa década, algumas iniciativas contribuíram para a organização do setor, como a estruturação da Secretaria Municipal de Saúde (SMS), em 1989, e a criação dos Distritos de Saúde (DS), os quais se consolidaram como unidades orçamentárias em meados da década de 1990 (Donnini, 2003; Neves, 2003).

Entre os anos de 1993 e 2000, o município vivenciou o Programa de Atenção à Saúde (PAS), período em que a estrutura da saúde pública foi quase toda desmantelada (Neves, 2003). A partir de 2000, a Secretaria Municipal de Saúde passou por nova reestruturação, que culminou na habilitação formal para a gestão plena da saúde do município (Neves, 2003), e implementou uma gestão democrática da saúde, voltada para atender mais efetivamente às suas demandas, sem perder a visão de conjunto da cidade na implantação de políticas públicas mais abrangentes. Na atual conformação, a Secretaria Municipal de Saúde dividiu o município em cinco coordenadorias de saúde: Norte, Sul, Leste, Sudeste e Centro-Oeste, e cada uma possui sua Supervisão Técnica de Saúde (STS), totalizando 25 supervisões técnicas de saúde.

As ações de Vigilância da Saúde passam a ser coordenadas pela Coordenação de Vigilância em Saúde, a qual engloba o controle de doenças e agravos, e o de zoonozes, a saúde ambiental e do trabalhador, a vigilância sanitária e a imunização (São Paulo, [s.d.]). 
Sabe-se que o modelo da Vigilância da Saúde é uma mudança paradigmática na forma de conceber e intervir no processo saúde-doença que só pode ser viabilizada por intermédio de propostas políticas e técnicas efetivas e de recursos humanos formados e capacitados para operar com base num 'novo' paradigma. Ou seja, esperava-se que a presença de professores e alunos de diferentes cursos de graduação vinculados à universidade pudesse impactar de forma positiva na construção de um novo paradigma. Esse deveria direcionar as práticas nas unidades de saúde na perspectiva de um modelo de Vigilância da Saúde inovador, o qual integrasse ações coletivas e planejamento participativo, assentando-se nas necessidades da coletividade e na intersetorialidade.

Fica evidente, nos discursos dos profissionais de saúde, que não têm sido realizadas discussões técnicas ou políticas relativas às mudanças no processo de trabalho. Nesse sentido, Rosa e Labate (2005) ressaltam a necessidade de formar um novo profissional para que ocorra uma mudança no modelo de atenção, salientando a mudança na formação dos profissionais de saúde como potencialidade para a transformação das práticas na realidade diária.

\section{Conclusões}

Foi possível constatar, assim como verificado por Feuerwerker, Costa e Rangel (2000), que a consolidação de práticas inovadoras nos cenários reais requer um passo fundamental: a transformação do processo de trabalho, sendo necessária a coerência entre o projeto dos serviços e a proposta educativa, em suas dimensões política, técnica e metodológica. Para tanto, as iniciativas de integração ensino-serviço devem envolver instâncias de gestão dos serviços de saúde, da universidade e da população.

Em se tratando de trabalhar sobre a realidade, transformar o processo de trabalho estabelecido e promover mudanças profundas nas relações, é preciso que o compromisso e a decisão de fazer parte do processo sejam institucionais, e não somente dos atores isolados.

Fica evidente, também, a necessidade de se continuar investindo no processo de construção de novas relações ensino-serviço-comunidade, sensibilizando-se gestores, universidades e lideranças comunitárias visando qualificá-las. Deve-se buscar ampliar as oportunidades e criar espaços efetivos de interlocução entre a universidade, os serviços, as lideranças comunitárias e os conselhos de saúde que levem à discussão do papel de todos esses atores na formação profissional e na reorganização da atenção básica à saúde.

O desenvolvimento de projetos de ensino que tomam por base a realidade da saúde no país e do SUS colabora em muito para a mudança no paradigma de formação dos profissionais de saúde, o que, em última instância, 
possibilita a formação de uma massa de profissionais capazes de viabilizar o modelo da Vigilância da Saúde dentro do SUS.

Em que pese a longa escassez de políticas públicas para a estruturação da Vigilância da Saúde, recentemente ganharam espaço no cenário brasileiro iniciativas dos ministérios da Saúde e da Educação com vistas a fortalecer a formação profissional para a construção desse novo agir em saúde.

A portaria $n .^{0} 3.252$, de 22 de dezembro de 2009 , assinala que

a Vigilância em Saúde tem como objetivo a análise permanente da situação de saúde da população, articulando-se num conjunto de ações que se destinam a controlar determinantes, riscos e danos à saúde de populações que vivem em determinados territórios, garantindo a integralidade da atenção, o que inclui tanto a abordagem individual como coletiva dos problemas de saúde (Brasil, 2009).

A mesma portaria define que são ações da Vigilância em Saúde: a promoção da saúde da população, a vigilância epidemiológica, a vigilância da situação de saúde, a vigilância em saúde ambiental, a vigilância da saúde do trabalhador e a vigilância sanitária.

Aponta que a Vigilância em Saúde, visando à integralidade do cuidado, deve inserir-se na construção das redes de atenção à saúde, coordenadas pela Atenção Primária à Saúde, bem como no cotidiano das equipes de Atenção Primária/Saúde da Família, com atribuições e responsabilidades definidas em território único de atuação, integrando os processos de trabalho, planejamento, programação, monitoramento e avaliação dessas ações (Brasil, 2009).

O Programa de Educação pelo Trabalho para a Saúde na área de Vigilância em Saúde (PET-Saúde/VS), instituído pela portaria conjunta n. ${ }^{\circ} 3$, de 3 de março de 2010, pelos ministérios da Saúde e da Educação, tem como objetivo fomentar a formação de grupos de aprendizagem tutorial na área de Vigilância da Saúde (Brasil, 2010). O PET-Saúde/VS consistirá em grupos de trabalho compostos de alunos de graduação da área da saúde, docentes de instituições de ensino superior e trabalhadores das unidades de Vigilância em Saúde dos municípios. É a primeira modalidade do PET temático, uma vez que no seu primeiro ano de execução, 2009, o PET-Saúde foi dirigido apenas para a atuação nas unidades de Saúde da Família (Brasil, 2010).

O programa tem como pressuposto a educação pelo trabalho, buscando a qualificação em serviço dos profissionais da saúde, bem como a iniciação ao trabalho e vivências direcionadas aos estudantes, de acordo com as necessidades do SUS (Brasil, 2010). A portaria ressalta como objetivos do programa, dentre outros, os de contribuir para a formação de profissionais de saúde com perfil adequado às necessidades e às políticas de saúde do país na área de vigilância em saúde; sensibilizar e preparar profissionais para o adequado enfrentamento das diferentes realidades de vida e de saúde da 
população brasileira; e fomentar o papel da vigilância em saúde na análise da situação de saúde como instrumento de gestão, articulando-se em um conjunto de ações que se destinam a controlar determinantes, riscos e danos à saúde de populações que vivem em determinados territórios (Brasil, 2010).

\section{Notas}

1 Enfermeira e professora da Faculdade de Enfermagem da Universidade Federal de Mato Grosso (UFMT), Cuiabá. Mato Grosso, Brasil. Mestre em Enfermagem em Saúde Coletiva pela Escola de Enfermagem da Universidade de São Paulo (EEUSP). $<$ juguisardi@yahoo.com.br> Correspondência: Rua Fernando Correia da Costa, 2.367, Bairro Boa Esperança, CEP 78060900, Cuiabá, Mato Grosso, Brasil.

2 Enfermeira e professora do Departamento de Saúde Coletiva na Escola de Enfermagem da Universidade de São Paulo (EEUSP), São Paulo, Brasil. Doutora em Enfermagem pela Escola de Enfermagem da Universidade de São Paulo. <lislaine@usp.br>

\section{Referências}

BERTOLOZZI, Rita Maria; FRACOLLI, Lislaine Aparecida. Vigilância à saúde: alerta continuado em saúde coletiva. O Mundo da Saúde, São Paulo, v. 28, n. 1, p.14-20, 2004.

BRASIL. Portaria conjunta n. ${ }^{\circ}$, de março de 2010. Institui, no âmbito do Programa de Educação pelo Trabalho para a Saúde (PETSaúde), o PET-Saúde/Vigilância em Saúde. Diário Oficial da União, Poder Executivo, Brasília, DF, Seção 1, p. 59, 3 mar. 2010.

Ministério da Saúde. Portaria n. ${ }^{\circ}$ 3.252, de 22 de dezembro de 2009. Aprova as diretrizes para execução e financiamento das ações de Vigilância em Saúde pela União, estados, Distrito Federal e municípios e dá outras providências. Disponível em: <www.brasilsus.com.br/legislacoes/gm/ 102068-3252>. Acesso em: 5 mar. 2010.

Ministério da Saúde. Secretaria de Gestão do Trabalho e da Educação na Saúde. Departamento de Gestão da Educação na Saúde. Aprender SUS: o SUS e os cursos de graduação da área da saúde. Brasília, DF: Ministério da Saúde, 2004.

CECCIM, Ricardo B.; FEUERWERKER, Laura. Mudança na graduação das profissões de saúde sob o eixo da integralidade. Cadernos de Saúde Pública, Rio de Janeiro, v. 20, n. 5, p. 1.400-1.410, 2002.

DONNINI, Osvaldo Antonio. A reorganização da gestão de saúde em São Paulo. In: 
SOUZA, Maria Fátima; MENDES, Aquilas. Tempos radicais da saúde em São Paulo: a construção do SUS na maior cidade brasileira. São Paulo: Hucitec, 2003. p. 31-73.

FARIA, Lilian Saldanha et al. Vigilância da saúde: reconhecendo diferentes proposições conceituais na literatura científica. Saúde e Sociedade, São Paulo, v. 14, supl. 1, p. 176, 2005.

FEUERWERKER, Laura; COSTA, Heloniza; RANGEL, Maria Ligia. Diversificação de cenários de ensino e trabalho sobre necessidade/problemas da comunidade. Divulgação em Saúde para Debate, Rio de Janeiro, n. 22, p. $36-48,2000$.

LEFRÈVE, Fernando; LEFRÈVE, Ana Maria Cavalcanti; TEIXEIRA, Jorge Juarez Vieira (org.). O discurso do sujeito coletivo: uma nova abordagem metodológica na pesquisa qualitativa. Caxias do Sul: Educs, 2000.

NEVES, Hélio. Vigilância em saúde na capital paulista. In: SOUZA, Maria Fátima; MENDES, Aquilas. Tempos radicais da saúde em São Paulo: a construção do SUS na maior cidade brasileira. São Paulo: Hucitec, 2003.
PEREIRA, Juliana Guisardi. Articulação ensino-serviço para a construção da Vigilância da Saúde: em foco o distrito Butantã. Dissertação (Mestrado em Enfermagem) - Escola de Enfermagem, Universidade de São Paulo, São Paulo, 2007.

ROSA, Walisete de Almeida Godinho; LABATE, Renata Curi. Programa Saúde da Família: a construção de um novo modelo de assistência. Revista Latino-Americana de Enfermagem, Ribeirão Preto, v. 13, n. 6, p. 1.027-1.034, nov./dez. 2005.

SÃO PAULO. Prefeitura do Município. Secretaria Municipal de Governo. Sumário de dados, 2004. São Paulo: Secretaria Municipal de Governo, [s.d.]. Disponível em: <www.capital.sp.gov.br>. 2005. Acesso em: 25 out. 2006.

TEIXEIRA, Carmen Fontes; PAIM, Jairnilson Silva; VILASBÔAS, Ana Luiza. SUS, modelos assistenciais e vigilância da saúde. Informe Epidemiológico do SUS, Brasília, $\mathrm{DF}$ v. 7, n. 2, p. 7-28, 1998.

Recebido em 17/03/2010

Aprovado em 28/10/2010 